\title{
Simulation of wind velocities on long span structures: a novel stochastic wave based model
}

\author{
Brett A. Benowitz ${ }^{\mathrm{a}, *}$, George Deodatis ${ }^{\mathrm{b}}$ \\ ${ }^{a}$ Applied Science Division, Weidlinger Associates, Inc., New York, NY, USA \\ ${ }^{b}$ Department of Civil Engineering and Engineering Mechanics, Columbia University,
} New York, NY, USA

\begin{abstract}
A methodology is presented for efficient and accurate modeling of correlated wind velocities along long span structures at a virtually infinite number of points. Currently, the standard approach is to model wind velocities as discrete components of a multivariate stochastic vector process, characterized by a Cross-Spectral Density Matrix. To simulate sample realizations of the vector process, the Spectral Representation Method is one of the most commonly used, which involves a Cholesky decomposition of the Cross-Spectral Density Matrix. However, it is a well known issue that as the length of the structure, and consequently the size of the vector process, increases, this Cholesky decomposition breaks down numerically. Alternatively, this paper introduces the use of the frequency-wavenumber spectrum to model the wind velocities as a stochastic "wave," continuous in both space and time. This allows the wind velocities to be modeled at a virtually infinite number of points along the length of the structure. In this paper, the relationship

\footnotetext{
${ }^{*}$ Corresponding author

Email addresses: brett.benowitz@wai.com (Brett A. Benowitz), deodatis@civil.columbia.edu (George Deodatis)
}

Preprint submitted to J. Wind Eng. Ind. Aerodyn.

September 19, 2015

(C) 2015. This manuscript version is made available under the Elsevier user license http://www.elsevier.com/open-access/userlicense/1.0/
\end{abstract}


between the Cross Spectral Density Matrix and the frequency-wavenumber spectrum is first examined. The frequency-wavenumber spectrum is then derived for wind velocities. Numerical examples are carried out demonstrating that the simulated wave samples exhibit the desired spectral and coherence characteristics. The efficiency of this method, specifically through the use of the Fast Fourier Transform, is also demonstrated.

Keywords: Monte Carlo simulation, Spectral Representation Method, Wind velocity fields, Stochastic waves, Frequency-wavenumber spectrum

\section{Introduction \& motivation}

2

For tall buildings and long bridges alike, wind loads are often the governing design loads in structural design. This is especially true in the case of long-span bridges, where the dynamic behavior under wind loading is likely to be the governing design condition. Consider the well known case of the Tacoma-Narrows bridge which failed spectacularly under dynamic wind loading: the aeroelastic flutter that eventually lead to its failure [1] would never be accounted for in a static analysis. In order to account for the randomness in the wind loading, this is often considered probabilistically [2]. To that end, Monte-Carlo simulation is typically used in practice. Sample realizations of wind velocity fields are generated, and the response of the bridge is calculated. Ensemble statistics can then be calculated from the responses.

Wind velocity fields are, by nature, a three dimensional vector field. That is, at any point $\mathbf{x}=(x, y, z)$ in space, the wind velocity is a vector with three components $\mathbf{u}(\mathbf{x}, t)=\left[u_{x}(\mathbf{x}, t), u_{y}(\mathbf{x}, t), u_{z}(\mathbf{x}, t)\right]^{T}$, corresponding to the three Euclidean dimensions. It should be noted, though, that for clar- 
ity and simplicity, a scalar wind velocity field is considered throughout the remainder of this paper along with its spatial variation in a single direction (i.e. horizontally along the length of the bridge deck, $x$ ). For a bridge, these simplifications are fairly reasonable. It is implied that the cross-correlation between velocity components is negligible. This is a quite common assumption in practice [3 6]. Research efforts looking at the effect of this assumption have found that although there is an observable correlation, the magnitude is negligible when compared to the overall uncertainty in the wind velocity [4, $7[10$.

The most widely used method, and standard practice, for generating wind velocities along the length of a bridge is to model the wind velocities as a multi-variate process. That is, the wind field is discretized into discrete components of a multi-variate vector process, $\mathbf{u}(t)=\left[u_{1}(t), \ldots, u_{j}(t), \ldots, u_{n}(t)\right]^{T}$. The $j^{\text {th }}$ component $\left(u_{j}(t)\right)$ of the vector $\mathbf{u}(t)$ corresponds to the wind velocity field $u(x, t)$ at $x_{j}$. Sample realizations are then simulated using a wide range of methodologies. The Spectral Representation Method (SRM) is one of the most commonly used today and will be considered in this paper. Simulation of sample realizations of the multi-variate processes within the SRM centers around the Cross-Spectral Density Matrix (CSDM) which is decomposed using usually Cholesky's decomposition [11 13]. Alternatively, a modal decomposition can be used [14-16]. It is well known, however, that for both modal and Cholesky decompositions, there is an issue when the number of components in the vector process becomes large (on the order of 20-30 points). These decompositions break down due to the fact that neighboring points are highly correlated and so, as the CSDM grows in size, it 
becomes increasingly close to singular.

Recent research efforts have centered on approximate techniques to simplify this decomposition. Some examples of this approach include the following non-exhaustive list. Yang, Chang, and Chang [17] developed an explicit form for the Cholesky decomposition under certain assumptions. In [18, Cao et. al. developed algebraic expressions to explicitly express the Cholesky decomposition. Li et. al. [19] proposed a simplified formulation, which breaks the $n$-variate vector process, with $n$ correlated components, into smaller sized independent processes. These smaller vector processes (or even univariate processes) avoid the issues of large dimensionality, and are more computationally efficient. In [20], Ding et. al. reduce the computational effort by limiting the number of Cholesky decompositions necessary. However, the decomposition itself is not circumvented. For seismic applications, rather than wind, Gao et. al. [21] proposed the use of the lagged coherency matrix rather than the CSDM, since it is smoothly varying in frequency, and can then be coupled with interpolation methods to reduce the need for Cholesky decompositions. All of these methods, however, involve approximations to some degree. Furthermore, the majority of these methods remain quite computationally intensive, with many decompositions necessary for each simulation.

In this work, an alternative formulation will be presented in which a virtually infinite number of spatial points can be simulated exactly. This is achieved by modeling the wind velocities as a continuous 'wave' in space-time rather than a discrete vector in space and continuous only in time. Furthermore, the sample generation is comparatively efficient due to use of the Fast 
Fourier Transform (FFT). This formulation was initially presented in [22], but is significantly expanded upon in this paper. This paper is organized as follows. Section 2 reviews the SRM for multi-variate processes. Section 3 derives the frequency wavenumber spectrum used to describe the stochastic wave and establishes its relationship to the conventional multi-variate description of wind velocities. Section 4 expands upon the discussion of Section 3 and provides a detailed discussion of the numerical implementation of simulating stochastic waves, specifically for the wind velocity problem. Finally, Section 5 carries out two numerical examples to show the proposed formulation's validity, effectiveness, and accuracy. It should be noted that all of the theory and examples in this work assume stationarity and homogeneity.

\section{Review of multi-variate stochastic process simulation based on the SRM}

When modeling wind velocities along a long span, one must consider both the stochastic temporal and spatial variabilities. In order to model the spatial variation, the wind velocity "field" is typically modeled as a vector process, with discrete components. In this review, let us consider only stationary and Gaussian vector processes, for simplicity. For the non-stationary case, a discussion can be found in [12]. For the non-Gaussian case, a discussion can be found in [23].

With that in mind, let us define the notion of a vector process. A vector 
process is made up of individual, discrete, random processes:

$$
\mathbf{X}(t)=\left[\begin{array}{c}
X_{1}(t) \\
X_{2}(t) \\
\vdots \\
X_{j}(t) \\
\vdots \\
X_{n}(t)
\end{array}\right]
$$

where $X_{j}(t)$ is component $j$ of the $n$-variate vector process. Individually, each component is a univariate stochastic process, which can be considered in the conventional sense. However, when considered as a whole, the correlation between different components is important. These correlations are modeled via the Cross Correlation Matrix $(\mathrm{CCM}), R_{j k}(\tau)$, or the Cross Spectral Density Matrix (CSDM), $S_{j k}(\omega)$. The cross-correlations and cross-spectra form Fourier transform pairs, just as in the stationary univariate case (i.e. Wiener-Khinchin theorem/transform [24 27]):

$$
\begin{aligned}
& S_{j k}(\omega)=\frac{1}{2 \pi} \int_{-\infty}^{\infty} R_{j k}(\tau) e^{-i \omega \tau} \mathrm{d} \tau \\
& R_{j k}(\tau)=\int_{-\infty}^{\infty} S_{j k}(\omega) e^{i \omega \tau} \mathrm{d} \omega
\end{aligned}
$$

89 Considering here the homogeneous case (i.e. when the statistical properties 90 do not vary spatially), the CSDM is typically constructed from a prescribed auto-spectrum, $\left.S_{j j}(\omega)\right)^{1}$ and a coherence model $\gamma(\omega, \xi)$ [28] as follows:

$$
S_{j k}(\omega, \xi)=\sqrt{S_{j j}(\omega) S_{k k}(\omega)} \cdot \gamma(\omega, \xi)
$$

\footnotetext{
${ }^{1}$ note: repeated indices here do not imply summation
} 
where $\xi$ is the separation distance between nodes $j$ and $k$. It should be noted that $S_{j j}(\omega)=S_{k k}(\omega)$ for every value of $j$ and $k$. The auto-spectrum, $S_{j j}$, and the coherence function, $\gamma$, are chosen for the problem at hand. There are a multitude of options in the literature for most practical problems. For wind problems, as considered in this work, the auto-spectrum can be chosen to be the Kaimal Spectrum [29] (among various available options) and the coherence model is typically chosen to be the Davenport coherence function [30].

With the CSDM known - either from prescribed properties or estimated from data - the SRM can be used to simulate sample realizations of the vector process. First, the CSDM is decomposed as:

$$
\mathbf{S}(\omega)=\mathbf{H}(\omega) \mathbf{H}^{T *}(\omega)
$$

where the superscript ${ }^{T}$ denotes the matrix transpose, and ${ }^{*}$ denotes complex conjugate. This can be thought of as a typical Cholesky decomposition, and thus $\mathbf{H}$ is lower triangular:

$$
\mathbf{H}(\omega)=\left[\begin{array}{ccccc}
H_{11}(\omega) & 0 & 0 & \cdots & 0 \\
H_{21}(\omega) & H_{22}(\omega) & 0 & \cdots & 0 \\
H_{31}(\omega) & H_{32}(\omega) & H_{33}(\omega) & \cdots & 0 \\
\vdots & \vdots & \vdots & \ddots & \vdots \\
H_{n 1}(\omega) & H_{n 2}(\omega) & H_{n 3}(\omega) & \cdots & H_{n n}(\omega)
\end{array}\right]
$$


108

$$
X_{j}(t)=2 \sum_{m=1}^{j} \sum_{l=0}^{N-1}\left|H_{j m}\left(\omega_{l}\right)\right| \sqrt{\Delta \omega} \cos \left(\omega_{l} t+\Theta_{j m}\left(\omega_{l}\right)+\phi_{m l}\right) ; j=1,2, \ldots, n
$$

where:

$$
\begin{aligned}
\omega_{l} & =l \Delta \omega, l=0,1, \ldots, N-1 \\
\Delta \omega & =\frac{\omega_{u}}{N} \\
\Theta_{j m}\left(\omega_{l}\right) & =\tan ^{-1}\left(\frac{\operatorname{Im}\left[H_{j m}\left(\omega_{l}\right)\right]}{\operatorname{Re}\left[H_{j m}\left(\omega_{l}\right)\right]}\right)
\end{aligned}
$$

109

where $\operatorname{Im}[\cdot]$ and $\operatorname{Re}[\cdot]$ denote the imaginary and real parts, respectively; and $\phi_{m l}$ represents independent random phase angles between $[0,2 \pi]$. In this multi-variate case, $\phi_{m l}$ is a $n \times N$ matrix: $\phi_{m l}$ can be thought of as $n$ independent series of length $N$. In the form shown in Eqn. (6), the generated samples are not ergodic, in contrast to the typical univariate SRM samples [31]. An alternative form in [12, 32] double-indexes the frequencies in order to overcome this limitation.

As was mentioned earlier, it is well known that the decomposition of Eqn. 4 breaks down as $\mathbf{S}(\omega)$ grows (or $n \rightarrow \infty)$. This is independent of the decomposition used (i.e. modal or Cholesky), due to the fact that as $n \rightarrow \infty, \Delta x \rightarrow 0$, and these neighboring points become highly correlated. This correlation causes $\mathbf{S}(\omega)$ to become increasingly closer to singular, and the decompositions break down numerically. Furthermore, it should be noted that $\mathbf{S}(\omega)$ needs to be decomposed at each frequency $\omega_{l}$, which quickly adds up in computational cost. 


\section{Modeling wind as a stochastic wave}

\subsection{Derivation of frequency-wavenumber spectrum}

In this section, we propose the use of the frequency-wavenumber spectrum, $S(\omega, \kappa)$, to model the wind velocities as a stochastic "wave" continuous in both space and time. This allows the wind velocities to be modeled at a virtualy infinite number of points along the length of the structure. The Cross-Spectral Density Matrix (CSDM), or auto-spectrum and coherence model, can be transformed to the frequency-wavenumber spectrum through the following integral expression [28]:

$$
S(\omega, \kappa)=\frac{1}{2 \pi} \int_{-\infty}^{\infty} S(\omega) \cdot \gamma(\xi, \omega) e^{i \kappa \xi} \mathrm{d} \xi
$$

where $S(\omega)$ is the auto-spectrum, $\gamma(\xi, \omega)$ is the coherence model, $\xi$ is the spatial separation distance, $\omega$ is the angular frequency, and $\kappa$ is the wavenumber. It should be pointed out here that wavenumber representations have been used previously [33, 34] in wind engineering. These works model the wind velocity field as a frozen field, and describe the time variation by advecting the frozen field with a mean wind speed. The frequency-wavenumber model proposed here does not follow this assumption.

Several choices exist for the auto-spectrum, $S(\omega)$, in Eqn. (8). Some of the more commonly used in practice include: von Karman's spectrum [35, 36], Davenport's spectrum [37, Kaimal's spectrum [29], Simiu and Scanlan's spectrum [4], and Solari and Piccardo's spectrum [38] which models the full co-spectral dependencies across components. In this study, the Kaimal spectrum (which is of the same functional form as Simiu and Scanlan's) will 
be used, as this is one of the classical expressions used in wind engineering. A second example in Section 5.3 will also return to the von Karman spectrum. The Kaimal two-sided spectrum is defined (using parameter definitions from [4]) as [29]:

$$
S_{K}(\omega)=\frac{1}{2} \cdot \frac{200}{2 \pi} \cdot u_{*}^{2} \cdot \frac{z}{U(z)} \cdot \frac{1}{\left[1+50 \frac{\omega z}{2 \pi U(z)}\right]^{\frac{5}{3}}}
$$

where $z$ is the height above ground, $U(z)$ is the average wind velocity at height $z$, and $u_{*}$ is the velocity friction component defined as:

$$
u_{*}=\frac{k U(z)}{\ln \left(\frac{z}{z_{0}}\right)}
$$

where $k$ is von Karman's constant $(k \cong 0.4)$, and $z_{0}$ is a parameter describing the ground roughness.

Similarly, there are several choices for the coherence model, $\gamma(\xi, \omega)$, in Eqn. (8). Davenport's coherence function [30] is among the classical expressions. Kristensen and Jenson [39] proposed a model based on Taylor's hypothesis. More recently, Krenk [40] proposed a coherence model to account for the observation that the normalized co-spectral densities do not go to unity at $\omega=0$. For the purposes of this study, the Davenport coherence function will be used, defined as [30]:

$$
\gamma(\xi, \omega)=e^{-\frac{\lambda \omega \xi}{2 \pi U(z)}}
$$

where $\lambda$ is a decay parameter, often chosen to be between 7 and 10. A second example, using Krenk's coherence function, will also be carried out in Section 5.3 . 
164 165

Combining Eqns. (8), (9), and (11), the frequency-wavenumber spectrum, $S(\omega, \kappa)$, can be expressed as:

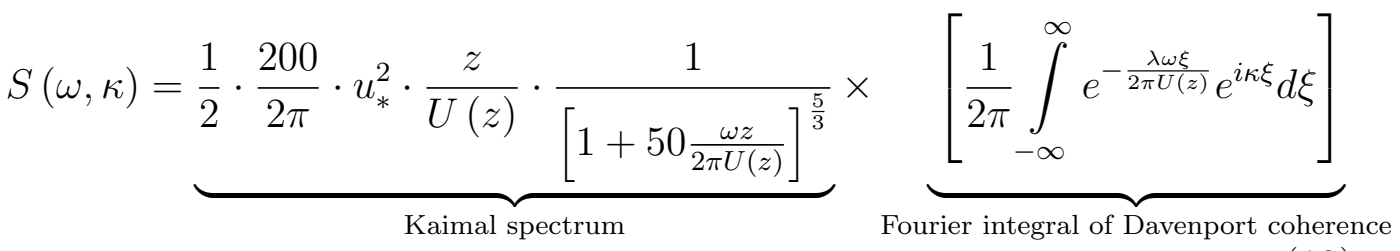

The Fourier integral in the second term has an analytical solution [41]. Therefore, Eqn. 12 simplifies to:

$$
S(\omega, \kappa)=\frac{1}{2} \cdot \frac{200}{2 \pi} \cdot u_{*}^{2} \cdot \frac{z}{U(z)} \cdot \frac{1}{\left[1+50 \frac{\omega z}{2 \pi U(z)}\right]^{\frac{5}{3}}} \times\left[\frac{\left(\frac{\lambda \omega}{\pi U(z)}\right)}{\kappa^{2}+\left(\frac{\lambda \omega}{2 \pi U(z)}\right)^{2}}\right]
$$

The frequency-wavenumber spectrum in Eqn. (13) is a generalized, closed form expression for modeling wind velocity fields in space-time as a stochastic wave. Sample realizations can be generated using the SRM formulation which will be discussed later in Section 4 .

\subsection{Verification of frequency-wavenumber spectrum}

In order for the frequency-wavenumber spectrum of Eqn. (13) to be valid, the spectral density $S(\omega)$, at any point in the spatial domain, should be the originally prescribed Kaimal spectrum, $S_{K}(\omega)$. From basic multi-variate probablity, any dimension of a multi-dimensional process can be integrated out to give the uni-variate spectral density in the remaining dimension(s) (e.g., [42]). Thus, for the two dimensional case of the wave being considered in this chapter:

$$
S(\omega)=\frac{1}{2 \pi} \int_{-\infty}^{\infty} S(\omega, \kappa) \mathrm{d} \kappa
$$


183

Here, the scaling of $\frac{1}{2 \pi}$ is added to be consistent with definitions used throughout this paper. For simplicity, a quantity $\rho(\omega)$ is defined, such that the frequency-wavenumber spectrum in Eqn. (13) can be rewritten as:

$$
S(\omega, \kappa)=S_{K}(\omega) \times\left[\frac{\rho(\omega)}{\kappa^{2}+\left(\frac{\rho(\omega)}{2}\right)^{2}}\right]
$$

where $\rho(\omega)$ is given by:

$$
\rho(\omega)=\frac{\lambda \omega}{\pi U(z)}
$$

Substituting Eqn. (15) into Eqn. (14), and solving:

$$
\begin{aligned}
S(\omega) & =\frac{1}{2 \pi} \int_{-\infty}^{\infty} S_{K}(\omega) \times\left[\frac{\rho(\omega)}{\kappa^{2}+\left(\frac{\rho(\omega)}{2}\right)^{2}}\right] \mathrm{d} \kappa \\
& =\frac{1}{2 \pi} S_{K}(\omega) \rho(\omega) \int_{-\infty}^{\infty} \frac{1}{\kappa^{2}+\left(\frac{\rho(\omega)}{2}\right)^{2}} \mathrm{~d} \kappa \\
& =\frac{1}{2 \pi} S_{K}(\omega) \rho(\omega) \times 2 \pi \sqrt{\frac{1}{\rho^{2}(\omega)}} \\
& =S_{K}(\omega)
\end{aligned}
$$

Therefore, it can be seen that at any point in the spatial domain, the autospectral characteristics are intact and still reflect the prescribed Kaimal spectrum.

It is also required that the coherence is retained throughout the spatial domain, however this is non-trivial to explore analytically. In Section 5 , the auto-spectral and coherence characeteristics of generated samples will be verified through numerical tests. 
192 94
195

\section{Simulation of wind velocities as a wave} (1) $u(x, t)=\sqrt{2} \sum_{l=1}^{N_{\kappa}} \sum_{m=1}^{N_{\omega}} \sum_{I_{\omega}= \pm 1} \sqrt{2 \cdot S_{f}\left(I_{\omega} \omega_{m}, \kappa_{l}\right) \cdot \Delta \omega \Delta \kappa} \cdot \cos \left[I_{\omega} \omega_{m} t+\kappa_{l} x+\phi_{m l}^{I_{\omega}}\right]$

where

$$
\begin{aligned}
\omega_{m} & =m \Delta \omega \\
\Delta \omega & =\frac{\omega_{u}}{N_{\omega}} \\
\kappa_{l} & =l \Delta \kappa \\
\Delta \kappa & =\frac{\kappa_{u}}{N_{\kappa}}
\end{aligned}
$$

However, the simulation becomes more efficient if a frequency shifting method is adopted, as proposed by Zerva [45]. In the standard SRM, the frequencies are discretized such that:

$$
\omega_{j}=j \Delta \omega
$$

Zerva [45] showed it is advantageous to shift the frequencies such that:

$$
\omega_{j}=\left(j+\frac{1}{2}\right) \Delta \omega
$$

There are several benefits to this formulation, as discussed in [45]. Worth noting are its (a) increased ensemble convergence rate, (b) simplified adaption to FFT, and (c) preservation of ergodicity properties. Specific to this example, note that there is a singularity at $S(\omega=0, \kappa=0)$ in Eqn. (13). 
This singularity is avoided by using this discretization, as the value for $S\left(\omega=\frac{\Delta \omega}{2}, \kappa=\frac{\Delta \kappa}{2}\right)$ is finite. In the case of a wave, both frequency, $\omega$, and wave-number, $\kappa$, are discretized in this matter. Using this discretization, the SRM formula of Eqn. (18) can be used directly.

Use of the FFT can dramatically increase the computational efficiency of simulation. In fact, the effects are compounded for this multi-dimensional case compared to the univariate, one-dimensional, stationary case. Efficiency gains of multiple orders of magnitude are thus possible. In order to make use of the FFT, Eqn. 18 is rearranged as:

$$
u(x, t)=\operatorname{Re}\left[\sum_{l=0}^{N_{\kappa}-1} \sum_{m=0}^{N_{\omega}-1}\left\{B_{l m}^{(1)} \exp \left[i \omega_{m} t+i \kappa_{l} x\right]+B_{l m}^{(2)} \exp \left[-i \omega_{m} t+i \kappa_{l} x\right]\right\}\right]
$$

where, $\operatorname{Re}[\cdot]$ denotes the real part, and:

$$
B_{l m}^{(n)}=2 \sqrt{S\left(\omega_{m}, \kappa_{n}\right) \cdot \Delta \omega \Delta \kappa} e^{i \phi_{l m}^{(n)}}
$$

Using a pseudo-notation for the FFT, Eqn. (22) can be re-written as a series of FFTs:

$$
u(x, t)=\operatorname{Re}\left\{\operatorname{FFT}_{\kappa}\left[\operatorname{FFT}_{\omega}\left(\mathbf{B}^{(1)}\right)\right]+\operatorname{FFT}_{\kappa}\left[\operatorname{IFFT}_{\omega}\left(\mathbf{B}^{(2)}\right)\right]\right\}
$$

where FFT (.) and IFFT $(\cdot)$ denote the Fast Fourier Transform and Inverse Fast Fourier Transform, respectively, and subscripts $\omega$ and $\kappa$ denote along which dimension the (I)FFT is computed.

Once the wave is simulated, time histories of individual points in space can be picked out, as necessary or desired. As a conceptual example, consider a bridge with a 1,000 meter main span. The designer builds a Finite Element model of the bridge with 10 meter segments representing the deck, and thus 


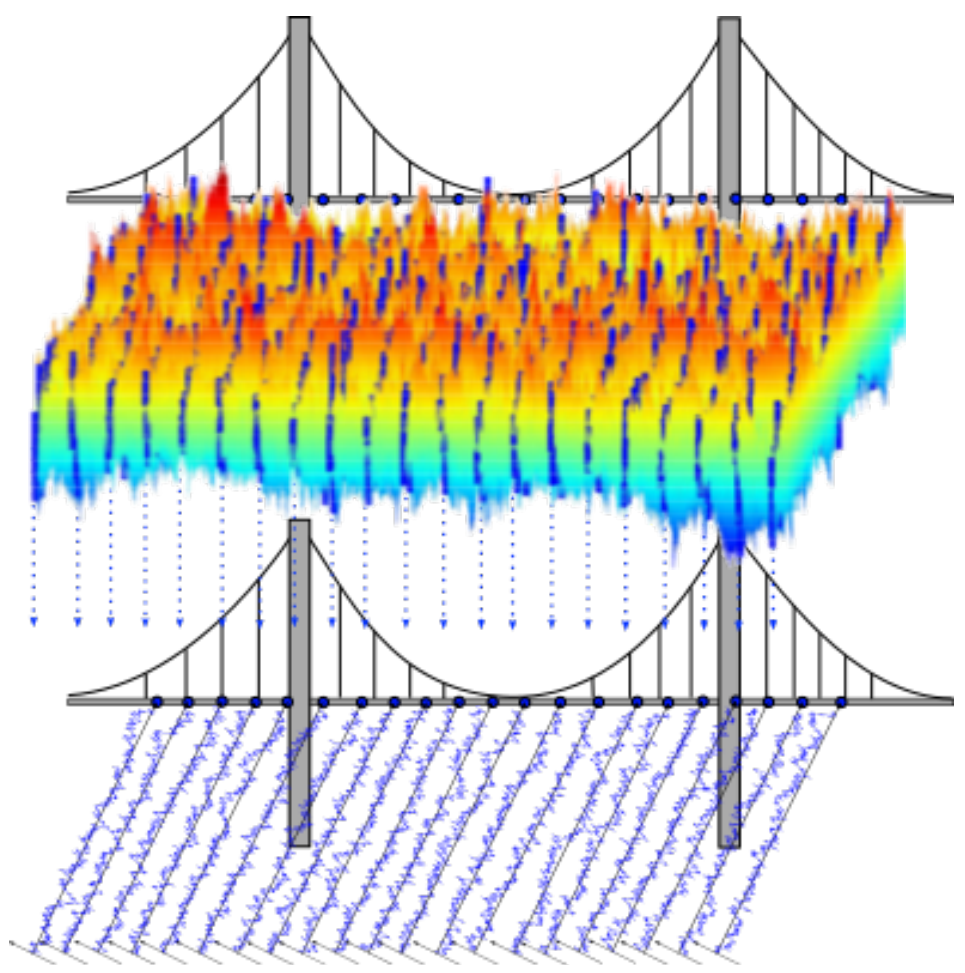

Figure 1: Simulation of wind velocities as a wave: The top image shows the simulated wave sample, and the bottom shows the individual "slices" that make up the time histories at each point of interest along the deck of the bridge

would like wind velocity time histories for each of the 100 nodes along the span. Using the conventional multi-variate methods, this would be extremely challenging or essentially impossible. In fact, those methods break down after about 20-30 points. Thus, the wind 'field' is modeled as a continuous wave as described above. With the continuous wave sample, the individual time histories at the 100 locations are simply the "slices" where $x$ is held constant, for each of the desired $x_{i}$. This is illustrated in Figure 1 . The continuous wave is shown on top, with slices highlighted in blue. These slices are then shown below as individual time histories. 


\section{Numerical examples}

In this section, two numerical examples are considered and the generated samples are tested to verify that their spectral characteristics match the prescribed auto-spectra and coherence functions. In other words, it is ensured that the samples simulated via the wave method and multi-variate method are statistically equivalent.

\subsection{Example 1: Problem definition}

The first example considered is a bridge with a 1600 meter long main span. The other parameter definitions are as follows:

- $L=1600$ m (main span length)

- $z=50.0 \mathrm{~m}$

- $z_{0}=0.03 \mathrm{~m}$

- $U(z)=40.0 \mathrm{~m} / \mathrm{s}$

- $\lambda=10.0$

Although the wind velocities are being modeled as a continuous wave, the wave is still discretized for numerical simulation. The frequency-wavenumber discretization used is:

- $\omega_{u}=8 \pi \mathrm{rad} / \mathrm{s}$

- $N_{\omega}=1,024$

- $\Delta \omega=\frac{\omega_{u}}{1023} \approx 0.0246 \mathrm{rad} / \mathrm{s}$ 
251

252

253

254

255

256

257

258

259

260

261

262

263

264

265

266

267

268

269

270

- $N_{\kappa}=16,368$

- $\kappa_{u}=\frac{2\left(N_{\kappa}-1\right)}{L} \pi=\frac{16367}{800} \pi \approx 64.273 \mathrm{~m}^{-1}$

- $\Delta \kappa=\frac{2 \pi}{L}=\frac{\pi}{800} \approx 0.0039 \mathrm{~m}^{-1}$

Due to the frequency shifting paradigm of [45] as described above, the uppercutoff frequency and wavenumber are actually shifted to:

- $\omega_{u}^{\prime}=\omega_{u}+\frac{\Delta \omega}{2} \approx 25.1450 \mathrm{rad} / \mathrm{s}$

- $\kappa_{u}^{\prime}=\kappa_{u}+\frac{\Delta \kappa}{2} \approx 64.2750 \mathrm{~m}^{-1}$

Time and space are discretized as follows:

- $N_{t}=2 N_{\omega}=2,048$

- $T=\frac{2 \pi}{\Delta \omega} \approx 255.6251 \mathrm{~s}$

- $\Delta t=\frac{T}{N_{t}}=\frac{\left(\frac{2 \pi}{\Delta \omega}\right)}{N_{t}} \approx 0.1249$

- $N_{x}=2 N_{\kappa}=32,736$

- $\Delta x=\frac{L}{N_{x}} \approx 0.0489 \mathrm{~m}$

There are some items in the discretization worth discussing. First, note that $N_{t}=2 N_{\omega}$ and $N_{x}=2 N_{\kappa}$. This is done because when using the FFT, the frequency is actually doubled and then padded with zeros. This is in accordance with the formulation in [44]. Also note that $N_{x}=32,736$. This high $N_{x}$ is chosen (a) to demonstrate the ability of this method to simulate a very high number of points in space, and (b) it was found through trial and error that this combination of $\kappa_{u}$ and $\Delta \kappa$ produced the best results - in 
271 272

terms of avoiding aliasing as well as closeness of fit to target coherence. All $N$ 's are chosen as powers of 2 to optimize the FFT efficiency. Finally, the specific relationship between frequency and time and space and wavenumber are in accordance with the MATLAB FFT routines which were used in this work.

\subsection{Example 1: Results}

In this numerical example, 5,000 samples are generated from the frequencywavenumber spectrum. At the start of the simulation, an index is chosen at random. In the results shown here, this index was $j=476$, for which $x_{j}=23.22 \mathrm{~m}$. The auto-spectrum was estimated at this location in space for each of the 5,000 samples, and the ensemble average of these auto-spectra is shown in Figure 2 compared with the prescribed Kaimal spectrum. For sample function 'slice' $u\left(t, x_{j}\right)$, the auto-spectrum is estimated from:

$$
\begin{aligned}
S_{j j}(\omega) & =\frac{\left|\int_{0}^{T} u\left(t, x_{j}\right) e^{-i \omega t} \mathrm{~d} t\right|^{2}}{2 \pi T} \\
& =\frac{\left|\mathrm{FFT}\left(\mathbf{u}^{(j)}\right)\right|^{2}}{\Delta \omega M_{\omega}^{2}}
\end{aligned}
$$

where $\operatorname{FFT}(\cdot)$ is following the notation defined earlier, $\mathbf{u}^{(j)}$ is a vector such that $u_{k}^{(j)}=u\left(t_{k}, x_{j}\right)$, and $M_{\omega}=2 N_{\omega}$ is the number of points used for the FFT, as described above.

The estimated auto-spectrum matches the target very nicely. Aside from very close to $\omega=0$, the two are indistinguishable. Thus, as was shown in Eqn. (17), the auto-spectral characteristics are retained in the wave model proposed. 


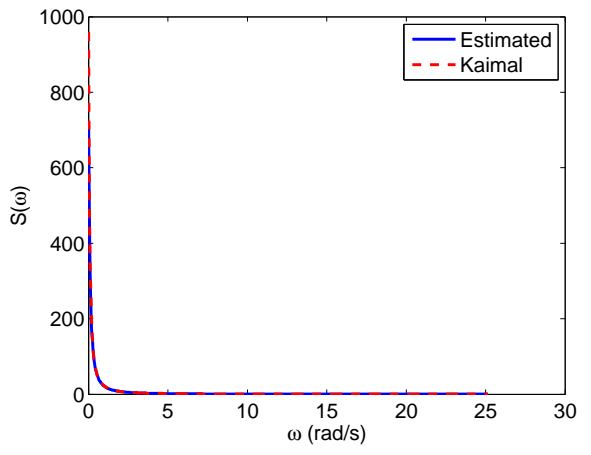

(a) Kaimal vs. estimated spectra

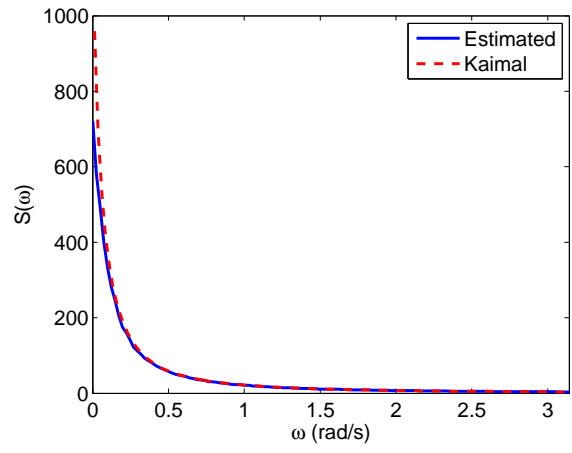

(b) Zoom-in detail

Figure 2: Comparison of the estimated spectra from 5000 generated samples using the wave model vs. the prescribed Kaimal auto-spectrum

Additionally, the cross-spectra are estimated for $S_{j(j+50)}(\omega), S_{j(j+100)}(\omega)$, and $S_{j(j+200)}(\omega)$. These indices correspond to $x_{526}=25.66 \mathrm{~m}, x_{576}=28.10$ $\mathrm{m}, x_{676}=32.99 \mathrm{~m}$. Therefore, the separation distances are: $\xi_{476,526}=2.44$ $\mathrm{m}, \xi_{476,576}=4.89 \mathrm{~m}, \xi_{476,676}=9.76 \mathrm{~m}$. For sample function slices $X\left(t, x_{j}\right)$ and $X\left(t, x_{k}\right)$, the cross-spectra are estimated from:

$$
\begin{aligned}
S_{j k}(\omega) & =\frac{\int_{0}^{T} u\left(t, x_{j}\right) e^{i \omega t} \mathrm{~d} t \int_{0}^{T} u\left(t, x_{k}\right) e^{-i \omega t} \mathrm{~d} t}{2 \pi T} \\
& =\frac{\operatorname{IFFT}\left(\mathbf{u}^{(j)}\right) \operatorname{FFT}\left(\mathbf{u}^{(k)}\right)}{\Delta \omega M_{\omega}^{2}}
\end{aligned}
$$

From these cross-spectra, the coherence functions are estimated as:

$$
\gamma\left(\omega, \xi_{j k}\right)=\frac{S_{j k}(\omega)}{S_{j j}(\omega) S_{k k}(\omega)}
$$

These three estimated coherence functions are plotted against the target Davenport coherence models for those specified separation distances in Figure 3. For all three, there is clear agreement between the target and the estimated. The first two are in fact perfectly aligned with the target. The 


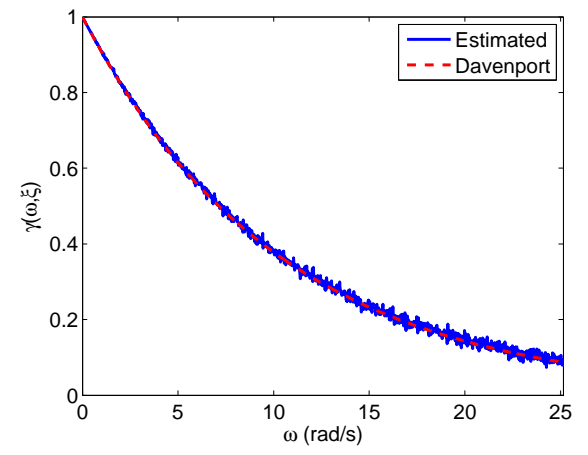

(a) $\gamma(\omega, \xi=2.44 \mathrm{~m})$

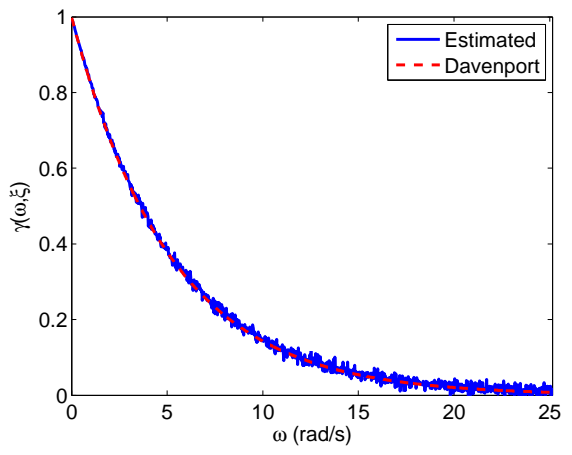

(b) $\gamma(\omega, \xi=4.89 \mathrm{~m})$

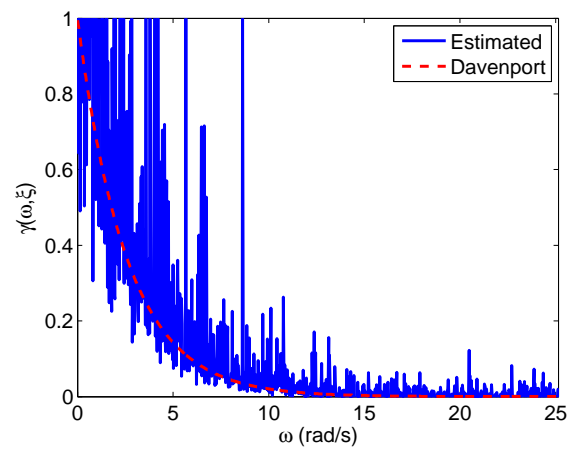

(c) $\gamma(\omega, \xi=9.78 \mathrm{~m})$

Figure 3: Comparison of the estimated coherence functions from 5000 generated samples using the wave model vs. the prescribed Davenport coherence function

third, for $\xi_{476,676}=9.78$ shown in Figure 3(c), though, is quite noisy. This is unfortunately inescapable. However, it is not an issue with the simulated samples, but rather with the estimation of the coherence and cross-spectra. It is well known that the coherence is very difficult to capture and is very sensitive [28], especially as the separation distance grows. These results are therefore perfectly acceptable.

A sample realization of the simulated wave is shown in Figure 4 . Figure 
296

4(a) shows the entire wave sample in space and time. Figure 4(b) then shows sample time histories taken at $x=0 \mathrm{~m}, x=800 \mathrm{~m}$, and $x=1600 \mathrm{~m}$. Lastly, Figure $4(\mathrm{c})$ shows zoom-in time histories at $x=23.22 \mathrm{~m}$ and $x=25.66$ $\mathrm{m}$, over-layed on top of each other to show the spatial variation between neighboring points in space.

Finally, the computational efficiency of the proposed algorithm is examined in Figure 5. This figure plots the CPU time (in seconds) to simulate a sample "wave" versus a discretization parameter, $N$. Note, that in this case $N_{\omega}=N_{\kappa}=N$ (i.e. there are $M^{2}=4 N^{2}$ points in the frequency-wavenumber domain). These CPU times were computed using MATLAB(R) R2013a, 64bit, on desktop workstation with a Intel(R) Core(TM) i7-2600 processor and 16GB RAM. For a problem of the scale of the above example, simulation of a single sample realization of the "wave" takes around 10 seconds. Note that this is independent of the number of spatial points considered, as they are just slices along the continuous wave. Using a multi-variate process simulation approach, this would take many hours, if even tractable.

\subsection{Example 2: Problem description}

In this second example, the von Karman auto-spectrum [35, 36] and Krenk coherence [40] will be used to demonstrate the flexibility of the proposed methodology. The example is similar to the second example in [46]. The von Karman auto-spectrum is defined as:

$$
S_{V K}(\omega)=\frac{4 L_{u} \sigma_{u}^{2}}{U(z)\left(1+70.8\left(\frac{\omega L_{u}}{2 \pi U(z)}\right)^{2}\right)^{5 / 6}}
$$



time
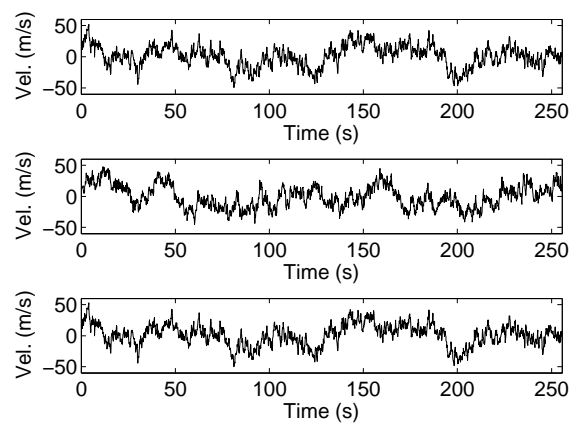

(b) Generated time histories. Top: $x=0$ m. Middle: $x=800 \mathrm{~m}$. Bottom: $x=$ $1600 \mathrm{~m}$.

Figure 4: Example 1: Generated sample realization of wind velocities modeled as a stochastic wave

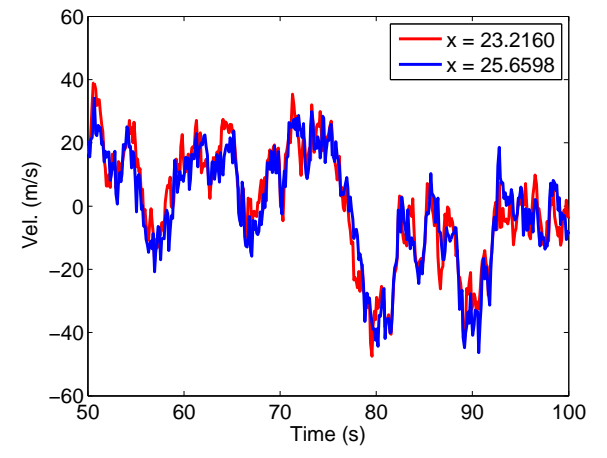

(c) Spatial variation between neighboring points 


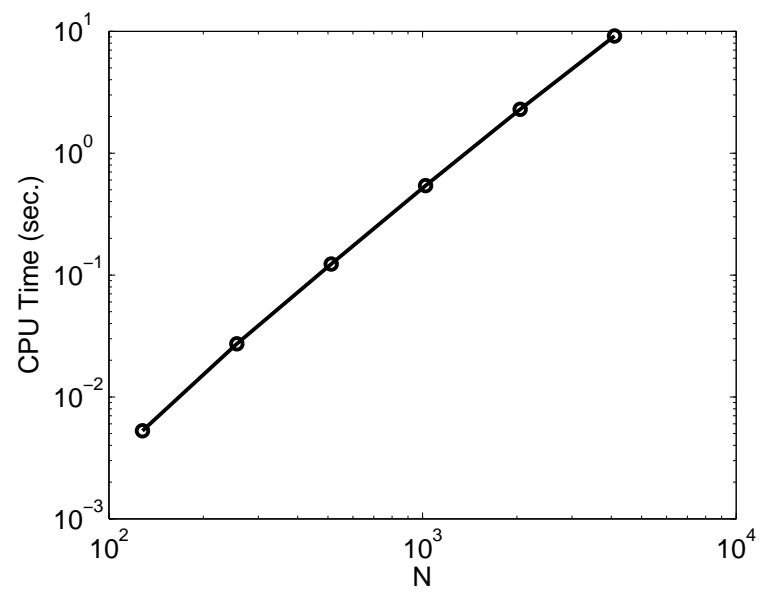

Figure 5: Computational efficiency of proposed methodology. CPU time in seconds vs. domain discretization, where $N_{\omega}=N_{\kappa}=N$.

where $L_{u}$ is the integral length scale, and $\sigma_{u}$ is the standard deviation of the turbulence. The Krenk coherence function is defined as:

$$
\gamma(\xi, \omega)=(1-0.35 \alpha \xi) e^{-0.7 \alpha \xi}
$$

where:

$$
\alpha=\sqrt{\left(\frac{\omega}{U(z)}\right)^{2}+\left(\frac{1}{l_{u}}\right)^{2}}
$$

and $l_{u}=1.34 L_{u}$.

Substituting Eqns. (28) and (29) into Eqn. (8) and taking the Fourier transform of the coherence term yields:

$$
S(\omega, \kappa)=\underbrace{\left[\frac{4 L_{u} \sigma_{u}^{2}}{U(z)\left(1+70.8\left(\frac{\omega L_{u}}{2 \pi U(z)}\right)^{2}\right)^{5 / 6}}\right]}_{\text {von Karman Spectrum }} \times \underbrace{\left[\frac{(0.7 \alpha)^{3}+2.1 \alpha \kappa^{2}}{2 \pi\left((0.7 \alpha)^{2}+\kappa^{2}\right)^{2}}\right]}_{\text {Fourier Integral of Krenk Coherence }}
$$


The parameter definitions for this example are as follows:

- $z=50 \mathrm{~m}$

- $L_{u}=150 \mathrm{~m}$

- $U(z)=35 \mathrm{~m} / \mathrm{s}$

- $\sigma_{u}=5 \mathrm{~m} / \mathrm{s}$

All other paramters were the same as in Example 1 (Section 5.1).

\subsection{Example 2: Results}

Once again, 5,000 samples are generated from the frequency-wavenumber spectrum. At the start of the simulation, an index is chosen at random. In the results shown here, this index was $j=26,221$, for which $x_{j}=1,281.5 \mathrm{~m}$.

The auto-spectrum is estimated at this location in space for each of the 5,000 samples, and the ensemble average of these auto-spectra is shown in Figure 6 compared with the prescribed von Karman spectrum. The auto-spectra are once again estimated using Eqn. (25). The estimated auto-spectrum again matches the target very nicely, with similar behavior to that in Example 1.

The cross-spectra are also again estimated, using Eqn. (26), for $(j, j+50)$, $(j, j+100)$, and $(j, j+200)$. These indices correspond to coordinates of $x_{26271}=1,284 \mathrm{~m}, x_{26321}=1,286.4 \mathrm{~m}$, and $x_{26421}=1291.3 \mathrm{~m}$ and separation distances of $\xi_{j, j+50}=2.44 \mathrm{~m}, \xi_{j, j+100}=4.89 \mathrm{~m}$, and $\xi_{j, j+200}=9.78 \mathrm{~m}$. From the cross-spectra, the coherence functions are again estimated using Eqn. (27). The estimated coherence functions for the three separation distances are plotted against the target Krenk coherence function in Figure 7. For all three, there is again clear agreement between the target and the estimated. 


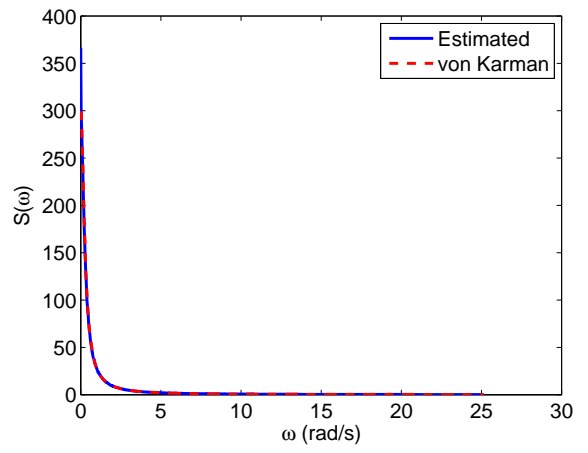

(a) von Karman vs. estimated spectra

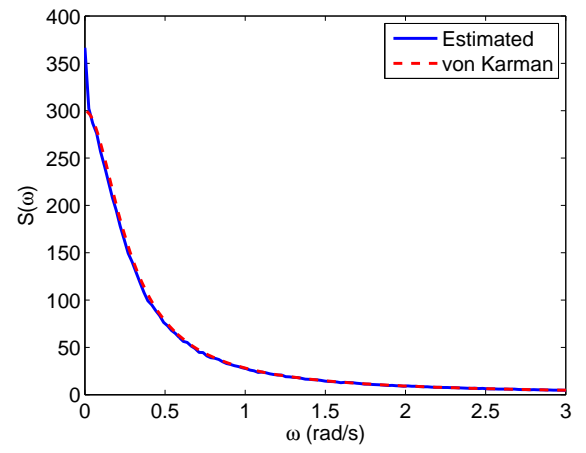

(b) Zoom-in detail

Figure 6: Comparison of the estimated spectra from 5000 generated samples using the wave model vs. the prescribed von Karman auto-spectrum

A similar behavior as in Example 1 is again witnessed, with noise increasing with separation distance. This is again an artifact of the estimation techniques. Note that the coherence at $\xi=9.78 \mathrm{~m}$ in Figure $7(\mathrm{c})$ actually becomes negative. This is the distinction of the Krenk coherence, which allows it to more appropriately characterize turbulence along long-span structures [40], by ensuring that the average turbulence integrates to zero.

Finally, a sample realization of the simulated wave is plotted in Figure 8. Figure 8(a) shows the entire wave sample in space and time. Figure 8(b) then shows sample time histories taken at $x=0 \mathrm{~m}, x=800 \mathrm{~m}$, and $x=1600$ m. Lastly, Figure 8(c) shows zoom-in time histories at $x=1,281.5 \mathrm{~m}$ and $x=1,284 \mathrm{~m}$, over-layed on top of each other to show the spatial variation between neighboring points in space. 


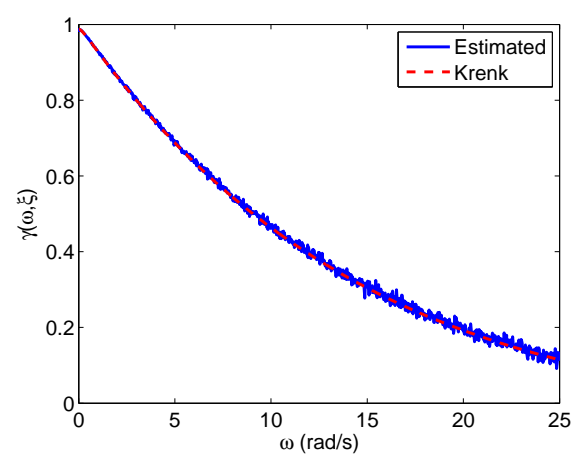

(a) $\gamma(\omega, \xi=2.44 \mathrm{~m})$

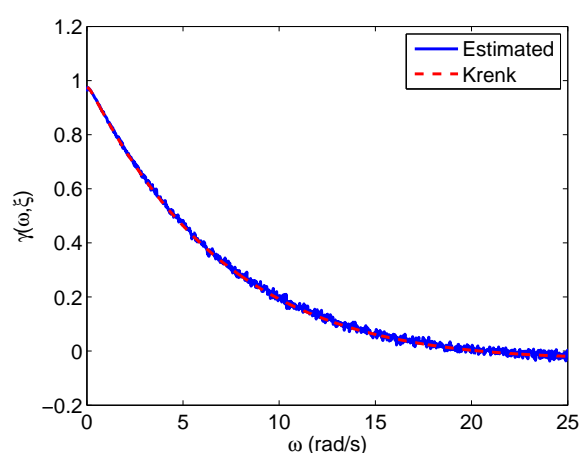

(b) $\gamma(\omega, \xi=4.89 \mathrm{~m})$

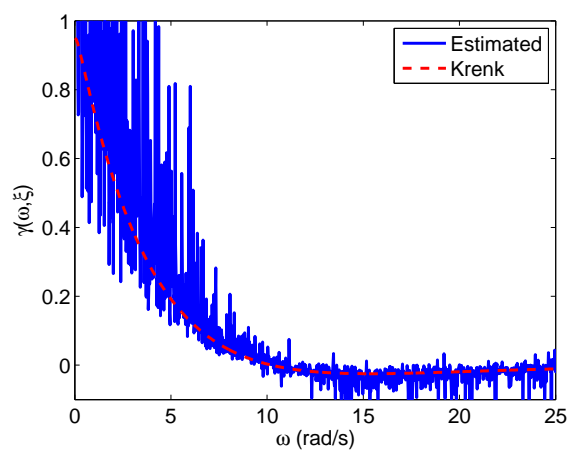

(c) $\gamma(\omega, \xi=9.78 \mathrm{~m})$

Figure 7: Comparison of the estimated coherence functions from 5000 generated samples using the wave model vs. the prescribed Krenk coherence function 

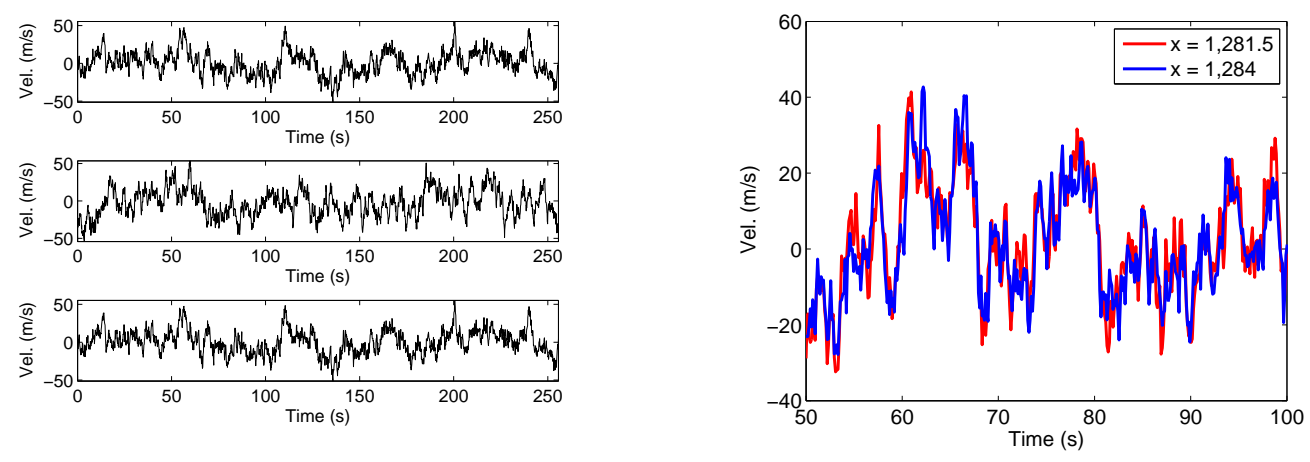

(b) Generated time histories. Top: $x=0$

(c) Spatial variation between neighboring m. Middle: $x=800 \mathrm{~m}$. Bottom: $x=$ points $1600 \mathrm{~m}$.

Figure 8: Example 2: Generated sample realization of wind velocities modeled as a stochastic wave 


\section{Conclusions}

A novel approach to simulating wind velocity time histories along longspan structures was presented. In contrast to the existing methodologies of modeling these wind velocities as stochastic vector processes, it was proposed to model them as a stochastic wave which is continuous in space and time. This allows the simulation of wind velocities at a virtually infinite number of points in space, a feat which was virtually impossible up to this point. Furthermore, the method was shown to be accurate, robust, and more efficient than previous methodologies.

This methodology could potentially be extended to other cases. The formulation in this paper was for one-dimensional, homogeneous in space, and stationary in time, velocity fields. Alternatively, each of these 'categories' could be extended to multi-dimensionality, non-homogeneity, and non-stationarity, respectively.

\section{Acknowledgments}

This work was supported in part by the National Science Foundation under IGERT Award \#DGE-0905597, Solving Urbanization Challenges By Design.

\section{References}

[1] K. Y. Billal and R. H. Scanlan. Resonance, Tacoma Narrows bridge failure, and undergraduate physics textbooks. American Journal of Physics, 59(2):118-124, 1991. 
[2] A. Davenport. The application of statistical concepts to the wind loading of structures. ICE Proceedings, (6480):449-472, 1961.

[3] X. Chen, M. Matsumoto, and A. Kareem. Aerodynamic coupling effects on flutter and buffeting of bridges. Journal of Engineering Mechanics, 126:17-26, 2000.

[4] E. Simiu and R. H. Scanlan. Wind Effects on Structures: An Introduction to Wind Engineering. John Wiley \& Sons, Inc., 1978.

[5] E. Strmmen. Theory of bridge aerodynamics. Springer, 2006.

[6] F. Tubino and G. Solari. Gust buffeting of long span bridges: double modal transformation and effective turbulence. Engineering Structures, 29:1698-1707, 2015.

[7] N. Minh, T. Miyata, H. Yamada, and Y. Sanada. Numerical simulation of wind turbulence of buffeting analysis of long-span bridges. Journal of Wind Engineering and Industrial Aerodynamics, 83:301-315, 1999.

[8] O. Øiseth, A. Rönnquist, and R. Sigbjörnsson. Simplified prediction of wind-induced response and stability limit of slender long-span suspension bridges, based on modified quasi-steady theory: a case study. Journal of wind engineering and industrial aerodynamics, 98(12):730$741,2010$.

[9] Wind cross-spectrum effects on long-span bridges, 1992. ASCE.

[10] O. Øiseth, A. Rönnquist, and R. Sigbjörnsson. Effects of co-spectral densities of atmospheric turbulence on the dynamic response of cable- 
supported bridges: A case study. Journal of Wind Engineering and Industrial Aerodynamics, 116:83-93, 2013.

[11] M. Shinozuka and C. Jan. Digital simulation of random processes and its applications. Journal of Sound and Vibrationon, 25(1):111-128, 1972.

[12] G. Deodatis. Non-stationary stochastic vector processes: seismic ground motion applications. Probabilistic Engineering Mechanics, 11(3):149167, jul 1996.

[13] G. Deodatis. Simulation of ergodic multivariate stochastic processes. Journal of Engineering Mechanics, 122(8):778-787, 1996.

[14] M. Shinozuka, C.-B. Yun, and H. Seya. Stochastic methods in wind engineering. Journal of Wind Engineering and Industrial Aerodynamics, 36:829-843, jan 1990.

[15] M. Di Paola. Digital simulation of wind field velocity. Journal of Wind Engineering and Industrial Aerodynamics, 74-76:91-109, apr 1998.

[16] M. Di Paola and I. Gullo. Digital generation of multivariate wind field processes. Probabilistic Engineering Mechanics, 16(1):1-10, jan 2001.

[17] W. Yang, T. Chang, and C. Chang. An efficient wind field simulation technique for bridges. Journal of Wind Engineering and Industrial Aerodynamics, 67-68:697-708, apr 1997.

[18] Y. Cao, H. Xiang, and Y. Zhou. Simulation of stochastic wind velocity field on long-span bridges. Journal of Engineering Mechanics, 126(1): $1-6,2000$. 
[19] Y. Li, H. Liao, and S. Qiang. Simplifying the simulation of stochastic wind velocity fields for long cable-stayed bridges. Computers \& Structures, 82(20-21):1591-1598, aug 2004.

[20] Q. Ding, L. Zhu, and H. Xiang. An efficient ergodic simulation of multivariate stochastic processes with spectral representation. Probabilistic Engineering Mechanics, 26(2):350-356, apr 2011.

[21] Y. Gao, Y. Wu, D. Li, H. Liu, and N. Zhang. An improved approximation for the spectral representation method in the simulation of spatially varying ground motions. Probabilistic Engineering Mechanics, 29:7-15, jul 2012.

[22] B. A. Benowitz and G. Deodatis. Simulation of wind velocities on long span structures: A novel stochastic wave based model. In 11th International Conference on Structural Safety and Reliability (ICOSSAR2013), pages 5549-5553. CRC Press / Balkema, 2013.

[23] M. D. Shields and G. Deodatis. A simple and efficient methodology to approximate a general non-gaussian stationary stochastic vector process by a translation process with applications in wind velocity simulation. Probabilistic Engineering Mechanics, 31:19-29, 2013.

[24] N. Wiener. Generalized harmonic analysis. Acta Mathematica, 55:117258, 1930.

[25] A. Khintchine. Korrelationstheorie der stationären stochastischen Prozesse. Mathematische Annalen, 109(1):604-615, dec 1934. 
[26] M. Priestley. Spectral Analysis and Times Series Volume 1: Univariate Series. Academic Press, 1981.

[27] A. Papoulis. Probability, Random Variables, and Stochastic Processes. WCB/McGraw-Hill, 3rd edition, 1991.

[28] A. Zerva. Spatial Variation of Seismic Ground Motions: Modeling and Engineering Applications. CRC Press, Taylor \& Francis Group, Boca Raton, FL, 2009.

[29] J. C. Kaimal, J. C. Wyngaard, Y. Izumi, and O. R. Coté. Spectral characteristics of surface-layer turbulence. Quarterly Journal of the Royal Meteorological Society, 98(417):563-589, jul 1972.

[30] A. Davenport. The dependence of wind loads on meteorological parameters. Proceedings of the International Research Seminar on Wind Effects on Buildings and Structures, 1:19-82, 1967.

[31] M. Shinozuka and G. Deodatis. Simulation of stochastic processes by spectral representation. Applied Mechanics Reviews, 44(4), 1991.

[32] M. Shinozuka, M. Kamata, and C.-B. Yun. Simulation of earthquake ground motion as multi-variate stochastic process. Technical report, Department of Civil Engineering and Operations Research, Princeton University, 1989.

[33] J. Mann and S. Krenk. Fourier simulation of a non-isotropic wind field model. In . Y. Schueller, Shinozuka, editor, Structural Safety \& Reliability, pages 1669-1674, 1994. 
[34] J. Mann. Wind field simulation. Probabilistic Engineering Mechanics, 13(4):269-282, oct 1998.

[35] T. von Karman. Progress in the statistical theory of turbulence. Proceedings of the National Academy of Sciences of the United States of America, 34:530-539, 1948.

[36] R. I. Harris. Some further thoughts on the spectrum of gustiness in strong winds. Journal of Wind Engineering and Industrial Aerodynamics, 33:461-477, 1990.

[37] A. G. Davenport. The spectrum of horizontal gustiness near the ground in high winds. Quarterly Journal of the Royal Meteorological Society, 87:194-211, 1961.

[38] G. Solari and G. Piccardo. Probabilistic 3-d turbulence modeling for gust buffeting of structures. Probabilistic Engineering Mechanics, 16: 73-86, 2001.

[39] L. Kristensen and N. O. Jenson. Lateral coherence in isotropic turbulence and in the natural wind. Boundary-Layer Meteorology, 17:353-373, 1979.

[40] S. Krenk. Wind field coherence and dynamic wind forces. In A. Naess and S. Krenk, editors, IUTAM Symposium on Advances in Nonlinear Stochastic Mechanics, pages 269-278, 1995.

[41] R. Haberman. Applied Partial Differential Equations with Fourier Series and Boundary Value Problems. Pearson Prentice Hall, Upper Saddle River, New Jersey, 4th edition, 2004. 
491

[42] D. Newland. An Introduction to Random Vibrations, Spectral 83 Wavelet Analysis. Longman Scientific \& Technical, Essex, England, 3rd edition, 1993.

[43] G. Deodatis and M. Shinozuka. Simulation of seismic ground motion using stochastic waves. Journal of engineering mechanics, 115(12):27232737, 1989 .

[44] M. Shinozuka and G. Deodatis. Stochastic wave models for stationary and homogeneous seismic ground motion. Structural Safety, 10:235-246, 1991.

[45] A. Zerva. Seismic ground motion simulations from a class of spatial variability models. Earthquake Engineering 83 Structural Dynamics, 21: 351-361, 1992.

[46] K. Aas-Jakobsen and E. Strømmen. Time domain buffeting response calculations of slender structures. Journal of Wind Engineering and Industrial Aerodynamics, 89:341-364, 2001. 